\title{
Updates in HER2-Positive and Triple-Negative Breast Cancers
}

\author{
Presented by Melinda L. Telli, MD, and William J. Gradishar, MD
}

\begin{abstract}
The recent approval of 4 agents for the treatment of patients with metastatic HER2-positive breast cancer has led to expanded recommendations in the NCCN Guidelines for treatment of this disease. For triple-negative disease, immunotherapy continues to gain traction in this challenging subtype, both in the preoperative and metastatic settings, though not yet as adjuvant treatment. Sacituzumab govitecan is another new agent with strong utility in triple-negative disease, and PARP inhibitors are recommended options in BRCA-mutated disease.
\end{abstract}

J Natl Compr Canc Netw 2021;19(5.5):605-609 doi: $10.6004 /$ jnccn.2021.5005

Patients with HER2-positive breast cancer can now benefit from 4 newly approved agents shown to increase time to progression and, in some cases, overall survival (OS), as described at the NCCN 2021 Virtual Annual Conference by Melinda L. Telli, MD, Associate Professor of Medicine, Stanford University School of Medicine; Director, Breast Cancer Program, Stanford Cancer Institute; and Associate Director, Stanford Women's Cancer Center. Strides have also been made in triple-negative breast cancer (TNBC), with the refinement of checkpoint inhibitors and the incorporation of other novel agents, as noted by William J. Gradishar, MD, the Betsy Bramsen Professor of Breast Oncology and Chief of Hematology/Oncology, Robert H. Lurie Comprehensive Cancer Center of Northwestern University, Chicago.

\section{Early HER2-Positive Disease: Movement Toward De-Escalation}

The NCCN Clinical Practice Guidelines in Oncology (NCCN Guidelines) for Breast Cancer reflect the excellent prognosis for patients with Tla and T1b, node-negative, HER2-positive disease with the recommendation for consideration of chemotherapy plus HER2-directed therapy in this group. ${ }^{1,2}$ Chemotherapy and HER2-directed therapy is indicated for patients with node-positive disease and tumors $>1 \mathrm{~cm}$. Importantly, all preferred regimens for preoperative and adjuvant treatment are now anthracycline-sparing.

The move to de-escalate treatment for low-risk, HER2positive disease started with the 2019 phase II APT trial of patients with tumors $\leq 3.0 \mathrm{~cm}$ and N0/N1mic disease. ${ }^{3}$ Treatment with weekly paclitaxel for 12 cycles plus trastuzumab for 1 year led to a 7 -year OS rate of 95\%, with only 4 of 406 patients experiencing distant recurrences. The subsequent ATEMPT trial evaluated the newer agent ado- trastuzumab emtansine (T-DM1) in stage 1 No/N1mic disease. ${ }^{4}$ Although 3-year disease-free survival was impressive at $97.7 \%$, toxicity was an issue, and therefore the NCCN Guidelines do not currently endorse the routine use of this regimen, Dr. Telli said.

Escalation of therapy is also important, for example in patients with residual disease after standard neoadjuvant therapy. In this setting, high-level evidence supports adjuvant T-DM1 based on a 3-year invasive disease-free survival of $88.3 \%$ in the KATHERINE trial, versus $77 \%$ with continued adjuvant trastuzumab therapy. 5 "This has become a standard and is endorsed in the NCCN Guidelines for early-stage disease," she said.

Building upon these studies, EA1181 CompassHER2pCR is now evaluating a de-escalated neoadjuvant approach with 12 weeks of taxane chemotherapy, trastuzumab, and pertuzumab in patients with stage II-IIIA disease who achieve a complete pathologic response (pCR; ClinicalTrials.gov identifier: NCT04266249). Furthermore, escalation is being evaluated in the AO11801 CompassHER2 Residual Disease study in which patients with residual disease will be randomized to T-DMl \pm tucatinib (NCT04457596).

"A lot of data is suggesting we can reduce the duration and amount of chemotherapy we are giving," Dr. Telli said. "In the future, we may be able to pull back even in stage II disease and in some stage III disease. Right now, I give a de-escalated regimen with paclitaxel + trastuzumab for patients with stage I node-negative disease."

\section{Four New Agents for Advanced HER2-Positive Disease}

Switching to a discussion of recurrent and stage IV disease, Dr. Telli indicated that taxane + trastuzumab and 
pertuzumab remains recommended in the first-line metastatic setting and T-DM1 in the second line. For the third line and beyond, 4 new agents have been recently approved: (1) tucatinib + capecitabine + trastuzumab, which is indicated after $\geq 1$ HER2-directed regimen for metastatic disease; (2) trastuzumab deruxtecan (T-DXd), which is indicated for use after $\geq 2$ HER2-directed regimens for metastatic disease; (3) margetuximab + chemotherapy, which is currently indicated after $\geq 2$ HER2directed regimens, at least one of which was for metastatic disease (OS data are pending); and (4) neratinib + capecitabine, which is indicated for use after $\geq 2$ HER2directed regimens for metastatic disease.

\section{Trastuzumab Deruxtecan and Tucatinib}

"We now have 2 great new options based on the DESTINYBreast01 and HER2CLIMB trials," Dr. Telli said (Table 1).

The open-label phase II DESTINY-Breast01 trial led to the approval of the potent antibody-drug conjugate TDXd, which was associated with a response rate of $60.9 \%$ and median progression-free survival (PFS) of 16.4 months. ${ }^{6}$ In an update presented at the 2020 San Antonio Breast Cancer Symposium, median PFS increased to 19.4 months and median OS to 24.6 months. " This is really impressive in such a heavily pretreated and refractory group, but although T-DXd is incredible in terms of efficacy, it does have a downside: pneumonitis and interstitial lung disease," she noted, adding that this complication has led to death in a few patients.

Tucatinib, an oral anti-HER2 tyrosine kinase inhibitor, was approved in 2020 after the phase III HER2CLIMB study of 615 patients met its prespecified efficacy boundary for OS $(P=.0074)$ at the first interim analysis. ${ }^{8}$ Risk of disease progression or death was reduced by $46 \%$ in the primary endpoint population $(P<.00001)$ and by $34 \%$ in the total population $(P=.00480)$. Median PFS was 7.8 versus 5.6 months for the tucatinib and placebo groups, respectively. Importantly, tucatinib also proved to be effective against brain metastases (active or stable), reducing the risk of central nervous system progression or death by $68 \%(P<.00001)$ and death by $42 \%(P=.005){ }^{9}$

\section{Margetuximab and Neratinib}

Margetuximab is a novel antibody designed to alter Fcy receptor affinities and increase affinity to variants of the CD16A receptor. The SOPHIA trial randomized 538 patients with 1 to 3 prior lines of therapy ( $\geq 2$ prior anti-HER2 therapies, including pertuzumab) to chemotherapy (capecitabine, eribulin, gemcitabine, or vinorelbine) plus margetuximab or trastuzumab. ${ }^{10}$ Median PFS was 5.8 months with margetuximab versus 4.9 months with trastuzumab (hazard ratio [HR], 0.76; $P=.033$ ). Benefit was greatest in patients with the CD16A Fc-gamma receptor allele.

\begin{tabular}{|lcc|}
\hline $\begin{array}{c}\text { Table 1. Side-By-Side Comparison of the DESTINY } \\
\text { and HER2CLIMB Trials }\end{array}$ & $\begin{array}{c}\text { DESTINY Breast-017 } \\
(\mathrm{n}=184)\end{array}$ & $\begin{array}{c}\text { HER2CLIMB } \\
(\mathrm{n}=612)\end{array}$ \\
\hline Median follow-up, mo & 20.5 & 14.0 \\
\hline Lines of prior therapy, median & 6 & 4 \\
\hline Prior TDM1, \% & 100 & 100 \\
\hline Prior trastuzumab, \% & 100 & 100 \\
\hline Prior pertuzumab, \% & 65.8 & 99.8 \\
\hline ORR (95\% Cl) & $61.4 \%$ & $40.6 \%$ \\
& $(54.0 \%-68.5 \%)$ & $(35.3 \%-46.0 \%)$ \\
\hline PFS, mo & 19.4 & 7.8 \\
\hline mOS, mo & 24.6 & 21.9 \\
\hline
\end{tabular}

Abbreviations: $m O S$, median overall survival; ORR, overall response rate; PFS, progression-free survival.

Neratinib, a pan-HER inhibitor, was evaluated in the phase III NALA trial of 662 women with $\geq 2$ lines of HER2directed therapy who were randomized to capecitabine + neratinib or lapatinib. ${ }^{11}$ PFS was improved with neratinib (HR, 0.76; $P=.0059$ ), while mean OS, the co-primary endpoint, was only numerically improved at 24.0 versus 22.2 months with lapatinib (HR, $0.88 ; P=.2086$ ).

\section{"The Million-Dollar Question"}

With so many third-line options, Dr. Telli asked, "How do you choose between T-DXd and tucatinib? That's the million-dollar question we are struggling with, and now we also have margetuximab and neratinib in the mix." Dr. Telli has been "thoroughly impressed" with T-DXd, but cannot ignore concerns about interstitial lung disease. "It is very important to respond quickly to pulmonary symptoms" by obtaining chest imaging, starting steroids, and discontinuing the drug upon any symptoms of pneumonitis, she emphasized.

For patients with brain metastases, the HER2CLIMB regimen of tucatinib/trastuzumab/capecitabine is an excellent option based on "clearly positive and impressive data," she added. The availability of subcutaneous trastuzumab in combination with the oral agents capecitabine and tucatinib provides patients with an alternative to intravenously administered therapy.

\section{TNBC: Does Platinum Add Value?}

In early-stage TNBC, anthracycline/taxane (AC-T) remains the backbone of neoadjuvant therapy. A question still being explored is whether the addition of a platinum drug to a foundational regimen of AC-T improves outcomes. In most trials, it does improve pCR; however, this does not always correlate with longer-term outcomes, Dr. Gradishar said. 
In GeparSixto, the addition of a platinum incrementally improved disease-free survival ( $\mathrm{HR}, 0.56 ; P=.0224$ ), although the foundational chemotherapy (paclitaxel + non-pegylated doxorubicin) may not have been optimal. ${ }^{12}$ In CALGB 40603, which used standard taxane followed by an anthracycline, the addition of platinum increased the pCR rate but not the event-free survival rate (HR, $0.84 ; P=.36) .{ }^{13}$ Additionally, a meta-analysis of a neoadjuvant trial incorporating a platinum found incremental improvement in pCR, no significant difference in event-free survival (HR, 0.72; $P=.094$ ) or OS (HR, 0.86; $P=.651)$, and an increase in toxicity. ${ }^{14}$

"Does a platinum add benefit or not? We have dueling trials with different results," Dr. Gradishar said. Therefore, the NCCN Breast Cancer Panel maintains that the inclusion of platinum is controversial (as noted in a footnote). Although they can be used, there is no compelling evidence to do so. This question may be answered in the ongoing NRG Br003 trial (ClinicalTrials.gov identifier: NCT02488967).

\section{Treatment of Residual Disease}

Preoperative therapy identifies patients with residual disease who may benefit from postoperative therapy. Based on the CREATE-X trial, capecitabine is a good option. ${ }^{15}$ Adjuvant capecitabine resulted in a $30 \%$ reduction in risk of recurrence, and therefore the panel considers its use justified in patients with residual TNBC after preoperative therapy.

Ongoing trials are continuing to explore the treatment of residual disease in patients with TNBC by comparing capecitabine and platinum (ECOG-ACRIN EA 1131; NCT02445391) and by studying pembrolizumab (SWOG 1418; NCT02954874). "These may further inform our recommendations for residual disease,” Dr. Gradishar said.

\section{Preoperative Immunotherapy for TNBC}

Based on its efficacy in the setting of metastatic TNBC, checkpoint inhibitors are being evaluated as add-ons to foundational chemotherapy in the preoperative setting. Three checkpoint inhibitors have increased pCR rates, especially in patients whose tumors express PD-L1. These drugs and their pivotal trial include durvalumab in GeparNuevo, ${ }^{16}$ pembrolizumab in KEYNOTE-522, ${ }^{17}$ and atezolizumab in IMpassion 031. ${ }^{18}$ Interestingly, atezolizumab did not convey benefit in the NeoTRIP trial. ${ }^{19}$

"There is a signal that adding a checkpoint inhibitor in TNBC-whether it is PD-L1-positive or PD-L1-negativemay impact the pCR rate," Dr. Gradishar said. "That's great, but does this translate into improvements in event-free survival and-hopefully, further downstream-OS?" The FDA's Oncologic Drugs Advisory Committee (ODAC) is paying attention to this disconnect. ODAC recently voted 10 to 0 to not support the approval of pembrolizumab + chemotherapy as neoadjuvant therapy for TNBC, despite the increase in pCR demonstrated in KEYNOTE-522. ${ }^{17}$ This is an indication that longer-term follow-up will be required before these regimens are approved, he said.

\section{Immunotherapy, Antibody-Drug Conjugates for Metastatic Disease}

"The NCCN Guidelines provide a laundry list of options in metastatic disease, but there are new additions, including checkpoint inhibitors for patients with PDL1-positive disease, PARP inhibitors for BRCA1/2 mutations, and sacituzumab govitecan," Dr. Gradishar said. Among breast cancer subtypes, TNBC is considered the most fitting for immunotherapy. Three pivotal trials have examined the benefit of checkpoint inhibitors in metastatic TNBC: atezolizumab/nab-paclitaxel in IMpassion $130,{ }^{20,21}$ pembrolizumab/chemotherapy in KEYNOTE$355,{ }^{22}$ and atezolizumab/paclitaxel in IMpassion131. ${ }^{23}$

In the IMpassion130 trial, which enrolled 902 patients, atezolizumab + nab-paclitaxel showed "fairly compelling evidence" for an improvement in PFS (HR, 0.63) and OS (HR, 0.71) in patients with PD-L1-positive disease; no benefit was seen in those with PD-L1-negative disease. ${ }^{20}$ In the final OS analysis, presented at the ESMO Virtual Congress 2020, median OS was 25.4 versus 17.9 months with nab-paclitaxel alone (HR, 0.67). ${ }^{21}$

KEYNOTE-355 randomized patients to chemotherapy (numerous options) \pm pembrolizumab and found a strong benefit in the $38 \%$ of patients with a PD-L1 composite positive score (CPS) of $\geq 10$. Median PFS was 9.7 months with pembrolizumab + chemotherapy versus 5.6 months with chemotherapy alone (HR, 0.65). ${ }^{22}$

"A PFS advantage is consistent across both these trials, which used different antibodies and a different scoring system (though identifying a similar fraction as PD-L1-positive). But OS benefit, at this point, has only been seen with atezolizumab," he noted.

The third trial, IMpassion131, which evaluated paclitaxel \pm atezolizumab, "was a fly in the ointment," as the results were negative for both the PD-L1-positive and overall population. ${ }^{23}$ Although the reasons are not clear, the clinical takeaway is that atezolizumab should be paired with nab-paclitaxel, not paclitaxel. Pembrolizumab can be paired with a taxane or carboplatin/gemcitabine, he said (Figure 1).

\section{Sacituzumab Govitecan}

The first-in-class Trop-2-directed antibody-drug conjugate sacituzumab govitecan has joined the NCCN Guidelines as an "other recommended regimen" for recurrent TNBC, based on the global phase III ASCENT trial that evaluated this drug versus physician's choice of chemotherapy. In data updated at ESMO 2020, treatment with sacituzumab govitecan yielded a median PFS of 5.6 
- Two randomized phase III trials confirm benefit of PFS, atezolizumab, and pembrolizumab approved by FDA

- OS data with atezolizumab shows 7.5-month improvement

- Await OS data with pembrolizumab

- Important to use companion diagnostic relevant to therapeutic agent (ie, SP-142 for atezolizumab; 22C3 CPS score $\geq 10$ for pembrolizumab)

- Cutoffs and thresholds still a work in progress

- Likely better biomarker of benefit than PD-L1; research is in progress

- Best chemotherapy backbone

- If using atezolizumab, data are only with nab-paclitaxel

- If using pembrolizumab, data suggest can use either taxane or carboplatin/gemcitabine

- Be aware of unique adverse effects, follow guidelines for immuno-oncology toxicity

Figure 1. Summary of immune checkpoint inhibitors in triple-negative breast cancer. Abbreviations: CPS, combined positive score; OS, overall survival; PFS, progression-free survival.

months versus 1.7 months with chemotherapy (HR, $0.41 ; P<.0001)$ and median OS of 12.1 vs 6.7 months, respectively (HR, $0.48 ; P<.0001){ }^{24}$

A recent exploratory analysis found sacituzumab govitecan benefits patients regardless of Trop-2 expression levels or germline $B R C A$ status, with the caveat that these were small patient subsets. ${ }^{25}$ Although neutropenia can be an adverse effect, growth factors can help manage this, he added.

\section{PARP Inhibitors in TNBC}

The NCCN Guidelines "call out" patients with BRCA mutations as candidates for 2 approved PARP inhibitors: olaparib and talazoparib, both with category 1 recommendations based on 2 phase III trials.

Olaparib was evaluated in the OlympiAD trial ${ }^{26}$ and talazoparib in the EMBRACA study, ${ }^{27}$ both compared with treatment of physician's choice. Both PARP inhibitors improved PFS in BRCA-mutated TNBC. In Olympi$\mathrm{AD}$, a $49 \%$ reduction in death was also observed in patients with no prior chemotherapy, and olaparib was effective in those with germline or somatic mutations in other DNA damage response pathway genes.

"One of the obvious questions arising from this is whether, in patients with TNBC and a germline BRCA mutation and a PD-L1-positive tumor, you can use a checkpoint inhibitor or PARP inhibitor. I'd probably start with chemotherapy and a checkpoint inhibitor, then go on to a PARP inhibitor," Dr. Gradishar offered.

Disclosures: Dr. Telli has disclosed serving as a scientific advisor for and receiving consulting fees from AbbVie, Inc., Celgene Corporation, Immunomedics, Inc., Merck \& Co., Inc., Lilly Oncology, Natera, and Pfizer Inc. Dr. Gradishar has disclosed serving as a scientific advisor for AstraZeneca Pharmaceuticals LP, MacroGenics, Inc., Pfizer Inc., Roche Laboratories, Inc./Genentech, Inc., and Seattle Genetics, Inc.

Correspondence: Melinda L. Telli, MD, Stanford Cancer Institute, 875 Blake Wilbur Drive, CC 2241, Stanford, CA 94305-5826.

Email: mtelli@stanford.edu; and

William J. Gradishar, MD, Robert H. Lurie Comprehensive Cancer Center of Northwestern University, 676 North St. Clair Street, Suite 850, Chicago, IL 60611. Email:w-gradishar@northwestern.edu

\section{References}

1. Gradishar W, Moran M, Abraham J, et al. NCCN Clinical Practice Guidelines in Oncology: Breast Cancer. Version 2.2021. Accessed March 12, 2021. To view the most recent version, visit NCCN.org

2. Fehrenbacher L, Capra A, Quesenberry $C$ Jr, et al. Distant invasive breast cancer recurrence risk in human epidermal growth factor receptor 2-positive T1a and T1b node-negative localized breast cancer diagnosed from 2000 to 2006: a cohort from an integrated health care delivery system. J Clin Oncol 2014;32:2151-2158.

3. Tolaney S, Guo H, Pernas S, et al. Seven-year follow-up analysis of adjuvant paclitaxel and trastuzumab trial for node-negative, human epidermal growth factor receptor 2-positive breast cancer. J Clin Oncol 2019;37:1868-1875.

4. Tolaney S, Trippa L, Barry W, et al. TBCRC 033: a randomized phase II study of adjuvant trastuzumab emtansine (T-DM1) vs paclitaxel in combination with trastuzumab for stage I HER2-positive breast cancer (ATEMPT) [abstract]. Presented at the 2019 San Antonio Breast Cancer Symposium; December 10-14, 2019; San Antonio, Texas. Abstract GS1-05.
5. Von Minckwitz G, Huang C, Mano M, et al. Trastuzumab emtansine for residual invasive HER2-positive breast cancer. N Engl J Med 2019;380:617-628.

6. Modi S, Saura C, Yamashita T. Trastuzumab deruxtecan in previously treated HER2-positive breast cancer. N Engl J Med 2020;382:610-621.

7. Modi S, Saura C, Yamashita T, et al. Updated results from DESTINYbreast01, a phase 2 trial of trastuzumab deruxtecan (T-DXd) in HER2 positive metastatic breast cancer [abstract]. Presented at the 2020 Virtual San Antonio Breast Cancer Symposium; December 8-11, 2020. Abstract PD3-06.

8. Murthy R, Loi S, Okines A, et al. Tucatinib, trastuzumab, and capecitabine for HER-2 positive metastatic breast cancer. N Engl J Med 2020;382:597-609.

9. Lin N, Borges V, Anders C, et al. Intracranial efficacy and survival with tucatinib plus trastuzumab and capecitabine for previously treated HER2positive breast cancer with brain metastases in the HER2CLIMB trial. J Clin Oncol 2020;38:2610-2619.

10. Rugo H, Im S, Cardoso F, et al. Phase 3 SOPHIA study of margetuximab + chemotherapy vs trastuzumab + chemotherapy in patients with 
HER2 + metastatic breast cancer after prior anti-HER2 therapies: second interim overall survival analysis [abstract]. Presented at the 2019 San Antonio Breast Cancer Symposium; December 10-14, 2019; San Antonio, Texas. Abstract GS1-02.

11. Saura C, Oliveira M, Feng $Y$, et al. Neratinib + capecitabine vs lapatinib + capecitabine in patients with HER2+ metastatic breast cancer previously treated with $\geq 2$ HER2-directed regimens: findings from the multinational, randomized, phase III NALA trial [abstract]. Presented at the 2019 ASCO Annual Meeting; May 31-June 4, 2019; Chicago, Illinois. Abstract 1002

12. Von Minckwitz G, Schneeweiss A, Loibl S, et al. Neoadjuvant carboplatin in patients with triple-negative and HER2-positive early breast cancer (GeparSixto; GBG 66): a randomized phase 2 trial. Lancet Oncol 2014;15:747-756

13. Sikov W, Berry D, Perou C, et al. Impact of the addition of carboplatin and/or bevacizumab to neoadjuvant once-per-week paclitaxel followed by dose-dense doxorubicin and cyclophosphamide on pathologic complete response rates in stage II to III triple-negative breast cancer: CALGB 40603 (Alliance). J Clin Oncol 2015;33:13-21.

14. Poggio F, Bruzzone M, Ceppi M, et al. Platinum-based neoadjuvant chemotherapy in triple-negative breast cancer: a systematic review and meta-analysis. Ann Oncol 2018;29:1497-1508.

15. Masuda N, Lee S, Ohtani S, et al. Adjuvant capecitabine for breast cancer after preoperative chemotherapy. N Engl J Med 2017;376:2147-159.

16. Loibl S, Untch M, Burchardi $\mathrm{N}$, et al. A randomised phase II study investigating durvalumab in addition to an anthracycline taxane-based neoadjuvant therapy in early triple-negative breast cancer: clinical results and biomarker analysis of GeparNuevo study. Ann Oncol 2019;30:1279-1288.

17. Schmid P, Cortes J, Pusztai L, et al. Pembrolizumab for early triplenegative breast cancer. N Engl J Med 2020;382:810-821.

18. Mittendorf $\mathrm{E}$, Zhang $\mathrm{H}$, Barrios $\mathrm{C}$, et al. Neoadjuvant atezolizumab in combination with sequential nab-paclitaxel and anthracycline-based chemotherapy versus placebo and chemotherapy in patients with early-stage triple-negative breast cancer (IMpassion031): a randomized, doubleblind, phase 3 trial. Lancet 2020;396:1090-1100

19. Gianni L, Huang C, Egle D, et al. Pathologic complete response to neoadjuvant treatment with or without atezolizumab in triple-negative, early high-risk and locally advanced breast cancer. NeoTRIPaPDL1
Michelangelo randomized study [abstract]. Presented at the 2019 San Antonio Breast Cancer Symposium; December 10-14, 2019; San Antonio, Texas. Abstract GS3-04.

20. Schmid P, Rugo H, Adams S; IMpassion130 Investigators. Atezolizumab plus nab-paclitaxel as first-line treatment for unresectable, locally advanced or metastatic triple-negative breast cancer (IMpassion130): updated efficacy results from a randomised, double-blind, placebocontrolled, phase 3 trial. Lancet Oncol 2020;21:44-59.

21. Ermens L, Adams S, Barrios C et al. IMpassion130: final overall survival analysis from the pivotal phase III study of atezolizumab plus nab-paclitaxel vs placebo plus nab-paclitaxel in previously untreated locally advanced or metastatic triple-negative breast cancer [abstract]. Presented at the ESMO Virtual Congress 2020; September 19-21, 2020. Abstract LBA16.

22. Cortes J, Cescon D, Rugo H, et al. Pembrolizumab plus chemotherapy versus placebo for previously untreated locally recurrent inoperable or metastatic triple-negative breast cancer (KEYNOTE-355): a randomized, placebo-controlled, double-blind, phase 3 clinical trial. Lancet 2020;396:1817-1828.

23. Miles D, Gilgorov J, Andre F, et al. Primary results from IMpassion131 a double-blind placebo-controlled randomized phase 3 trial of first-line paclitaxel plus atezolizumab for unresectable locally advanced/metastatic triple-negative breast cancer [abstract]. Presented at the ESMO Virtual Congress 2020; September 19-21, 2020. Abstract LBA15.

24. Bardia A, Tolaney S, Loirat D, et al. ASCENT: a randomized phase III study of sacituzumab govitecan vs treatment of physician's choice in patients with previously treated metastatic triple-negative breast cancer [abstract]. Presented at the ESMO Virtual Congress 2020; September 19-21, 2020. Abstract LBA17.

25. Hurvitz S, Tolaney S, Punie K. Biomarker evaluation in the phase 2 AS CENT study of sacituzumab govitecan versus chemotherapy in patients with metastatic triple-negative breast cancer [abstract]. Presented at the 2020 Virtual San Antonio Breast Cancer Symposium; December 8-11, 2020. Abstract GS3-06.

26. Robson M, Im S, Senkus E. Olaparib for metastatic breast cancer in patients with a germline BRCA mutation. N Engl J Med 2017;377:523-533.

27. Litton J, Rugo H, Ettl J, et al. Talazoparib in patients with advanced breast cancer and a germline BRCA mutation. N Engl J Med 2018:379:753-763.

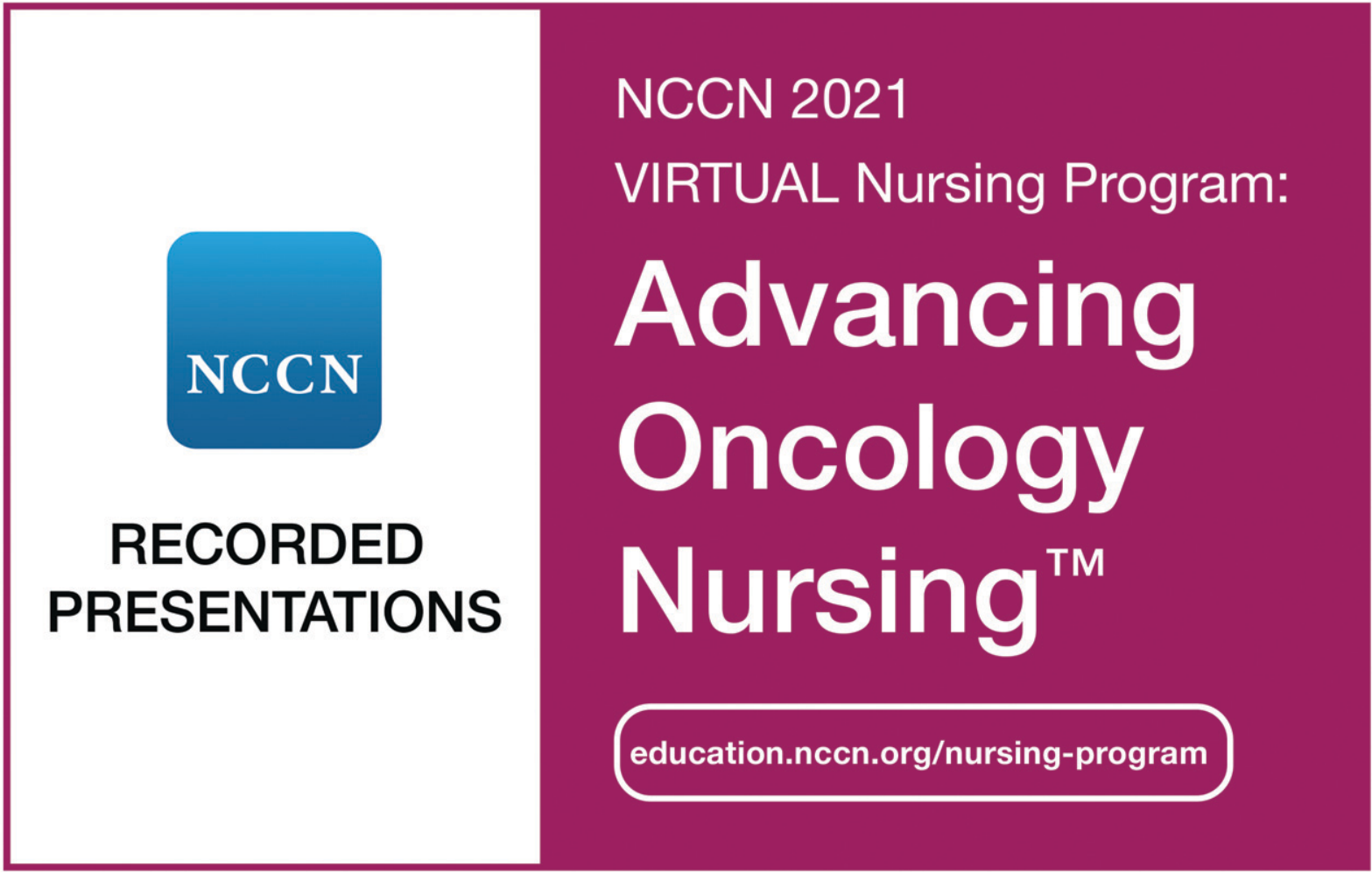

